\title{
Nonanalytic Magnetization Dependence of the Magnon Effective Mass in Itinerant Quantum Ferromagnets
}

\author{
D. Belitz \\ Department of Physics and Materials Science Institute, University of Oregon,Eugene, OR 97403 \\ T. R. Kirkpatrick \\ Institute for Physical Science and Technology, and Department of Physics \\ University of Maryland, College Park, MD 20742 \\ A. J. Millis \\ Department of Physics and Astronomy, The Johns Hopkins University \\ 3400 North Charles Street, Baltimore, MD 21218 \\ Thomas Vojta \\ Department of Physics and Materials Science Institute, University of Oregon, Eugene, OR 97403 \\ and Institut für Physik, TU Chemnitz-Zwickau, D-09107 Chemnitz, FRG
}

(April 24, 2021)

\begin{abstract}
The spin wave dispersion relation in both clean and disordered itinerant quantum ferromagnets is calculated. It is found that effects akin to weak-localization physics cause the frequency of the spin-waves to be a nonanalytic function of the magnetization $m$. For low frequencies $\Omega$, small wavevectors $\mathbf{k}$, and $m \rightarrow 0$, the dispersion relation is found to be of the form $\Omega=$ const. $\times m^{1-\alpha} \mathbf{k}^{2}$, with $\alpha=(4-d) / 2(2<d<4)$ for disordered systems, and $\alpha=(3-d)(1<d<3)$ for clean ones. In $d=4$ (disordered) and $d=3$ (clean), $\Omega \propto m \ln (1 / m) \mathbf{k}^{2}$. Experiments to test these predictions are proposed.
\end{abstract}

PACS numbers: 75.30.Ds; 75.40.Gb; 71.27.+a

One of the best known examples of quantum longrange order is the ferromagnetic (FM) state in itinerant electron systems at zero temperature $(T=0)$. An important manifestation of this order is the existence of spin waves [1]. In conventional Heisenberg ferromagnets the damping of the spin wave is negligible, and the dispersion relation has the form [2],

$$
\Omega=D(m) \mathbf{k}^{2}+o\left(|\mathbf{k}|^{2}\right)
$$

with $o(\epsilon)$ denoting terms that are smaller than $\epsilon$. The coefficient $D$ depends on the dimensionless magnetization $m=\left(n_{\uparrow}-n_{\downarrow}\right) / n$, with $n_{\uparrow}$ and $n_{\downarrow}$ the densities of spin-up and spin-down electrons, respectively, and $n=n_{\uparrow}+n_{\downarrow}$. In the conventional theory for clean 'weak ferromagnets' [2], $D(m \rightarrow 0)=D_{0} m . D_{0} \approx v_{\mathrm{F}} / k_{\mathrm{F}}$, with $k_{\mathrm{F}}$ the Fermi wavenumber and $v_{\mathrm{F}}=k_{\mathrm{F}} / \mu$ the Fermi velocity, is on the order of the inverse of the electron mass $\mu$, and Eq. (11) is valid for $|\mathbf{k}|<k_{\mathrm{F}} m \ll k_{\mathrm{F}}$. We will show below that these results do not correctly describe the small- $m$ behavior of metallic ferromagnets.

A crucial assumption in the derivation of Eq. (11) is that the interactions between spin fluctuations are shortranged. This assumption is of doubtful validity in the context of itinerant ferromagnets, since in metals at $T=0$ there exist soft modes that can couple to the spin fluctuations and lead to an effective long-ranged interaction. Indeed, recent work on the $T=0 \mathrm{FM}$ phase transition in both disordered [3] and clean [4] itinerant electron systems has shown that in spatial dimensions $d=2,3$ the asymptotic critical behavior is largely determined by the coupling of non-critical soft modes to the critical spin fluctuations. In disordered systems, these soft modes are the same 'diffusons' that cause the socalled weak-localization effects $[5]$. In clean systems, they are the usual particle-hole excitations that lead to the well-known nonanalyticities in Fermi liquids [6] which have recently been shown to be the clean analogues of the weak-localization effects [7]. These non-critical soft modes cause the critical spin fluctuations to interact via dimensionality dependent long-range effective forces. In the paramagnetic phase, the same physics is known to lead to a nonanalyticity in the wavenumber dependent spin susceptibility of the form

$$
\chi_{s}(\mathbf{k}) \sim \text { const. }+|\mathbf{k}|^{\zeta},
$$

with $\zeta=d-2$ (disordered) and $\zeta=d-1$ (clean), respectively [8].

In this Letter we consider the FM phase, and show that the long-ranged spin interactions that are mediated by the diffusons, or their clean counterparts, render invalid the standard results for the magnon dispersion. We find that a non-zero magnetization cuts off the long-ranged interaction at a scale $\ell_{m} \sim m^{-1}$ (clean) or $\ell_{m} \sim m^{-1 / 2}$ (disordered), which transforms the singular dependence on the wavenumber into one on the magnetization. The magnon dispersion is then given by Eq. (1), but with a nonanalytic $m$-dependence of $D$. For disordered electronic systems, we find 
$D(m \rightarrow 0)=c_{d} m\left[m^{-(4-d) / 2}+O(1)\right],(2<d<4)$

$D(m \rightarrow 0)=c_{4} m[\ln (1 / m)+O(1)],(d=4)$,

and $D(m \rightarrow 0) \sim m$ for $d>4$. For clean systems,

$$
\begin{aligned}
& D(m \rightarrow 0)=\tilde{c}_{d} m\left[m^{-(3-d)}+O(1)\right],(1<d<3), \\
& D(m \rightarrow 0)=\tilde{c}_{3} m[\ln (1 / m)+O(1)],(d=3) \quad, \quad(3 \mathrm{~b})
\end{aligned}
$$

and $D(m \rightarrow 0) \sim m$ for $d>3$. In these equations, $c_{d}$ and $\tilde{c}_{d}$ are positive constants.

In the remainder of this Letter we derive and further discuss these results. For simplicity, we consider a $d$-dimensional continuum model of interacting clean or disordered electrons [9], and pay particular attention to the particle-hole spin-triplet contribution to the electronelectron interaction term in the action, whose (repulsive) coupling constant we denote by $\Gamma_{t}$. Writing only the latter interaction term explicitly, and denoting the spin density by $\mathbf{n}_{s}$, the action reads

$$
S=S_{0}+S_{\text {int }}^{t}=S_{0}+\frac{\Gamma_{t}}{2} \int d x \mathbf{n}_{s}(x) \cdot \mathbf{n}_{s}(x),
$$

where $S_{0}$ contains all contributions to the action other than $S_{\text {int }}^{t}$. In particular, it contains the particle-hole spinsinglet and particle-particle interactions, as well as the coupling to the disorder. $\int d x \equiv \int d \mathbf{x} \int_{0}^{1 / T} d \tau$, and we use a $(d+1)$-vector notation $x \equiv(\mathbf{x}, \tau)$, with $\mathbf{x}$ a vector in real space, and $\tau$ the imaginary time. We perform a Hubbard-Stratonovich decoupling of $S_{\text {int }}^{t}$ by introducing a classical vector field $\mathbf{M}(x)$ with components $M_{i}$ $(i=1,2,3)$ that couples to $\mathbf{n}_{s}(x)$ and whose average is proportional to the magnetization, and we integrate out all fermionic degrees of freedom [10]. In this way we obtain the partition function in the form

$$
Z=e^{-F_{0} / T} \int D[\mathbf{M}] \exp [-\Phi(\mathbf{M})]
$$

Here $F_{0}$ is the part of the free energy that does not depend on the magnetization, and $\Phi$ is a Landau-GinzburgWilson (LGW) functional,

$$
\begin{aligned}
\Phi(\mathbf{M})= & \frac{\Gamma_{t}}{2} \int d x \mathbf{M}(x) \cdot \mathbf{M}(x) \\
& -\ln \left\langle\exp \left[-\Gamma_{t} \int d x \mathbf{M}(x) \cdot \mathbf{n}_{s}(x)\right]\right\rangle_{S_{0}}
\end{aligned}
$$

where $\langle\ldots\rangle_{S_{0}}$ denotes an average taken with respect to the reference action $S_{0}$.

Next, we expand in fluctuations about the ordered state. In order to ensure that the $O(3)$ symmetry is still manifest in the ordered state, we write 11]

$$
\mathbf{M}(x)=\rho(x) \hat{\phi}(x),
$$

with $\rho(x)$ the amplitude of $\mathbf{M}(x)$, and $\hat{\phi}(x)$ a unit vector,

$$
\hat{\phi}^{2}(x)=1
$$

Further, we take the system to be ordered in the 3direction and parametrize $\hat{\phi}$ and $\rho$ by

$$
\hat{\boldsymbol{\phi}}=(\boldsymbol{\pi}, \sigma)
$$

with $\boldsymbol{\pi}=\left(\pi_{1}, \pi_{2}\right), \sigma^{2}=1-\boldsymbol{\pi}^{2}$, and

$$
\rho(x)=m+\delta \rho(x),
$$

with $m=\langle\rho(x)\rangle$ proportional to the magnetization. According to Goldstone's theorem, the transverse fluctuations $\boldsymbol{\pi}(x)$ are soft, or of long range. $\Phi(\mathbf{M})$ can then be expanded in the fluctuations $\delta \rho$ and $\boldsymbol{\pi}$ as,

$$
\Phi(\mathbf{M})=\Phi\left(m \phi_{3}\right)+\delta \Phi(\mathbf{M})
$$

with $\phi_{3}$ a unit vector in 3-direction, and

$$
\begin{aligned}
\delta \Phi(\mathbf{M})= & \frac{\Gamma_{t}}{2} \int d x\left[\rho^{2}(x)-m^{2}\right] \\
& -\log \left\langle e^{-\Gamma_{t} \int d x \mathbf{M}(x) \cdot \mathbf{n}_{s}(x)-m n_{s, 3}(x)}\right\rangle_{S_{0}^{\prime}},
\end{aligned}
$$

with

$$
S_{0}^{\prime}=S_{0}-\Gamma_{t} m \int d x n_{s, 3}(x)
$$

The correlation functions in Eq. (8b) that one obtains by expanding the exponential determine the coefficients in the LGW functional. They are correlation functions of a reference ensemble whose action is given by Eq. (80), which describes the reference ensemble $S_{0}$ in an external magnetic field given by $-\Gamma_{t} m$. Here we are interested in the transverse spin susceptibility, which can be obtained from the imaginary frequency correlation function,

$$
\chi_{t}\left(\mathbf{k}, \Omega_{n}\right)=\left\langle\left|\pi_{1}\left(\mathbf{k}, \Omega_{n}\right)\right|^{2}\right\rangle \quad,
$$

with $\Omega_{n}$ a bosonic Matsubara frequency. Let us first consider the terms in Eq. (8b) that are bilinear in $\boldsymbol{\pi}$, which we denote by $\delta \Phi_{\pi \pi}$. We further integrate out $\rho(x)$ in saddle-point approximation, i.e. we neglect the fluctuations $\delta \rho$. We will justify this procedure later, and also discuss terms of higher order in $\boldsymbol{\pi}$. Taylor expanding Eq. (8b) gives,

$$
\delta \Phi_{\pi \pi}=\frac{\Gamma_{t} m}{2} \int d x d y \sum_{i, j=1}^{2} \pi_{i}(x) K_{i j}(x, y) \pi_{j}(y)
$$

with,

$$
\begin{aligned}
K_{i j}(x, y)= & -\Gamma_{t} m\left\langle n_{s, i}(x) n_{s, j}(y)\right\rangle_{S_{0}^{\prime}} \\
& -\delta(x-y) \delta_{i j}\left\langle n_{s, 3}(x)\right\rangle_{S_{0}^{\prime}}
\end{aligned}
$$

In this approximation, 
$\chi_{t}\left(\mathbf{k}, \Omega_{n}\right)=\frac{K_{11}\left(\mathbf{k}, \Omega_{n}\right)}{\Gamma_{t} m}\left[K_{11}^{2}\left(\mathbf{k}, \Omega_{n}\right)-K_{12}^{2}\left(\mathbf{k}, \Omega_{n}\right)\right]^{-1}$,

i.e., the kernel $K_{i j}$ determines the spin wave spectrum. Note the Goldstone mode structure of this result: Taking the Fourier transform of Eq. (10b), we have $(i=1,2)$

$$
\begin{gathered}
K_{i j}\left(\mathbf{k}, \Omega_{n}\right)=-\Gamma_{t} m \chi_{i j}^{(\mathrm{ref})}\left(\mathbf{k}, \Omega_{n}\right)-\delta_{i j}\left\langle n_{s, 3}\right\rangle_{S_{0}^{\prime}} . \\
\chi_{i j}^{(\mathrm{ref})}\left(\mathbf{k}, \Omega_{n}\right)=\left\langle n_{s, i}\left(\mathbf{k}, \Omega_{n}\right) n_{s, j}\left(-\mathbf{k},-\Omega_{n}\right)\right\rangle_{S_{0}^{\prime}},
\end{gathered}
$$

is the transverse part of the spin susceptibility in the reference ensemble with action $S_{0}^{\prime}$. A Ward identity that relates the reference system's magnetization to its static, homogeneous transverse spin susceptibility [1] ensures that $K_{i j}(0,0)=0$, i.e., transverse excitations are soft.

Expanding in powers of the frequency, one finds

$$
\chi_{i j}^{(\mathrm{ref})}\left(\mathbf{k}, \Omega_{n}\right)=\delta_{i j} \chi_{t}^{(\mathrm{ref})}(\mathbf{k})-\frac{i c}{m}\left|\Omega_{n}\right|\left[\delta_{i 1} \delta_{j 2}+\delta_{i 2} \delta_{j 1}\right],
$$

with $c \propto \mu^{2} / k_{\mathrm{F}}$ a constant. In the absence of weaklocalization effects, one would have $\chi_{t}^{(\mathrm{ref})}(\mathbf{k})=\chi_{t}-\tilde{c} \mathbf{k}^{2}$, with $\tilde{c}$ another constant independent of $m$. However, due to weak-localization effects in disordered systems, and their analogues in clean ones, $\chi_{t}^{(\mathrm{ref})}$ has a singularity at $\mathbf{k}=m=0$. For $m \equiv 0$ this has been shown using perturbation theory [8] as well as more general renormalization group (RG) arguments [7, 12]. It has also been shown that weak-localization effects (their clean counterparts) can be related to corrections to scaling near a disordered (clean) Fermi liquid fixed point [7]. Let us generalize those considerations to include the effects of a small magnetic field. The scale dimension of $\chi_{t}$ is zero [7], so in terms of a scaling function $F$ we have

$$
\chi_{t}^{(\mathrm{ref})}\left(\mathbf{k}, \ell_{m}^{-1}, u\right)=F\left(b \mathbf{k}, \ell_{m}^{-1} b, u b^{[u]}\right),
$$

with $\ell_{m}$ the magnetic length. The latter is determined perturbatively as follows. A nonzero magnetization leads to a mass or frequency cutoff in the soft modes that is given by a cyclotron frequency $\Omega_{c}$ with $m$ playing the role of the magnetic field. In clean (disordered) systems, the wavenumber scales like $\Omega\left(\Omega^{1 / 2}\right)$. Scaling the wavenumber with $\ell_{m}$ thus leads to $\ell_{m} \sim 1 / m$ in clean systems, and $\ell_{m} \sim 1 / \sqrt{m}$ in disordered ones. $u$ represents the leading irrelevant variable near the fixed point. Its scale dimension, $[u]$, is equal to $[u]=-(d-2)$ in disordered systems, and $[u]=-(d-1)$ in clean ones [7,8]. $b$ is a $\mathrm{RG}$ length rescaling factor. In the paramagnetic phase, $\ell_{m}^{-1}=0$, and Eq. (12a), with $b \sim|\mathbf{k}|^{-1}$, yields,

$$
\chi_{t}^{(\mathrm{ref})}(\mathbf{k}, 0, u) \sim \chi_{t}-c|\mathbf{k}|^{-[u]},
$$

with $c \sim u$. This is the nonanalyticity that leads to longrange interactions between the spin flucuations near the FM phase transition. For $\ell_{m}^{-1} \neq 0, \chi_{t}^{(\mathrm{ref})}$ is an analytic function of $\mathrm{k}^{2}$ and Eqs. (12a $12 \mathrm{~b}$ ) give

$$
\chi_{t}^{(\mathrm{ref})}\left(\mathbf{k}, \ell_{m}^{-1}, u\right) \sim \chi_{t}-c^{\prime}(m) \mathbf{k}^{2},
$$

with

$$
c^{\prime}(m) \sim \ell_{m}^{2+[u]} \sim\left\{\begin{array}{ll}
m^{-(2+[u]) / 2} & \text { (disordered) } \\
m^{-(2+[u])} & \text { (clean) }
\end{array} .\right.
$$

From this, with Eqs. (10) and (11), we immediately obtain our main results, Eqs. (3) (except for the nature of the leading correction terms in Eq. (11), which we will discuss below). Note that for disordered systems the dimensionless parameter characterizing 'small' wave numbers is $|\mathbf{k}| \ell \ll 1$, with $\ell$ the diffusive or transport mean-free path. The prefactors in Eqs. (3) are hard to estimate, since they depend on the value $\Gamma_{t}^{\text {ref }}$ of $\Gamma_{t}$ in the fully renormalized reference ensemble 12]. For instance, for the clean case in $d=3$ one finds, using the result of Ref. [8], $\tilde{c}_{3}=(32 \pi / 27)\left(N_{\mathrm{F}} \Gamma_{t}^{\mathrm{ref}}\right)^{2} / \mu$. Finally, we note that, at the level of the above scaling argument, the analyticity of $\chi_{t}^{(\mathrm{ref})}$ in powers of $\mathbf{k}^{2}$ for $\ell_{m}^{-1} \neq 0$ is an assumption. We have checked this explicitly in perturbation theory, verifying Eqs. (12d,12d) using both a $Q$-matrix field theory [7], and standard many-body perturbation theory, and will further discuss it from a RG point of view next.

We now show that the corrections to Eqs. (10) that result from taking into account the $\delta \rho$-fluctuations, as well as terms of higher than Gaussian order in $\boldsymbol{\pi}$, cannot change the above results. This is most easily done in the framework of the RG. We assign scale dimensions -1 and $-z$ to lengths and times, respectively, with $z$ the dynamical critical exponent, and scale dimensions $\left[\pi_{i}(x)\right]=\left(d+z-2+\eta^{\prime}\right) / 2$ and $[\rho(x)]=(d+z+\eta) / 2$ to the fields. Then Eq. (10a) tells us that there is a Gaussian fixed point with exponents

$$
\eta=2 \quad, \quad \eta^{\prime}=0 \quad, \quad z=2,
$$

that describes a FM state. To check for relevant operators that would destroy this fixed point, we systematically expand Eq. (8b) in powers of $\delta \rho$ and $\boldsymbol{\pi}$, and integrate out $\delta \rho$ perturbatively to obtain an effective action in terms of $\boldsymbol{\pi}$. There are several terms that dimensionally could lead to a $|\mathbf{k}|^{d-1}$ in the clean case and a $|\mathbf{k}|^{d-2}$ in the disordered case in Eq. (12d), rather than a $\mathbf{k}^{2}$ with a coefficient that is nonanalytic in $m$. In RG language, this would be a relevant operator with respect to our Gaussian fixed point. However, it turns out that there are Ward identities 11] that ensure, order by order in the expansion in fluctuations of the order parameter, that all terms of $O\left(\boldsymbol{\pi}^{2}\right)$, whether or not they couple to $\delta \rho$, are multiplied by at least a gradient squared [13]. We have also checked this by means of explicit perturbative calculations for selected vertices. Similar arguments show that 
the second term on the r.h.s. of Eq. (11) is the leading frequency dependence. As a result, the Gaussian fixed point identified above is stable by power counting. The leading nonanalytic correction to the $\Omega \sim \mathbf{k}^{2}$ dispersion arises from renormalizations of the Gaussian action due to terms of $O\left(\boldsymbol{\pi}^{4}\right)$. The resulting operators potentially have scale dimensions $2-d$ (disordered) and $d-1$ (clean), respectively. This reflects the largest possible corrections due to potentially soft modes; explicit calculations would be necessary to ascertain whether or not terms of this order actually exist [13]. We conclude that the Eqs. (12) are asymptotically exact. The exact magnon dispersion relation is thus given by Eq. (11), and the largest possible corrections are of $O\left(|\mathbf{k}|^{2+\zeta}\right)$, with $\zeta$ from Eq. (2)).

At $T>0$, temperature effects will compete with the magnetization in protecting the weak-localization singularities, and their clean counterparts, in the spin-triplet channel [14]. Therefore, for $m<T \ll T_{\mathrm{F}}$ in appropriate units, the $m$ in the brackets in Eqs. (3) will be replaced by $T$, leading to a nonanalytic $T$-dependence of the coefficient in the dispersion relation. Other consequences of a non-zero temperature are more subtle because of the occurrence of multiple temperature scales [4], and will be investigated separately in the future.

We conclude by discussing ways to experimentally verify our predictions. To our knowledge, no systematic studies of the prefactor of the $\mathbf{k}^{2}$-term in the dispersion relation have been performed. Such a study should be easier to do for disordered systems than for clean ones, since (1) the predicted effect is much larger in the disordered case, and (2) in the disordered case it will be easier to find a material near the FM quantum phase transition (e.g. by fine tuning the concentration of the magnetic ingredient in an alloy).

The most convincing experimental evidence would be an explicit measurement of the $m$-dependence of the dispersion relation. This would require measuring different samples with different values of the magnetization $m$, and extracting the $m$-dependence from the measured inverse magnon masses $D(m)$. In a $3-d$ disordered system, $D(m)$ for small $m$ should scale like $m^{1 / 2}$ (instead of $m$ according to RPA-like theories). Another possibility is to measure a single sample with a small magnetization, and to identify a quantitative difference of the measured magnon mass from that predicted by RPA-like theories. For instance, it has been reported that in $\mathrm{Fe}$ and $\mathrm{Ni}$ that prefactor is larger than expected by a factor of 2 to 3 15. Since the magnetization in these materials is not small, it is unlikely that this discrepancy is related to the predicted effect. However, similar experiments on materials with a small magnetization should suffice to corroborate or refute the present theory.

This work was initiated at the Aspen Center for Physics, and supported by the NSF under grant numbers DMR-95-10185, DMR-96-32978, and DMR-97-07701 and by the DFG under grant number Vo659/1-1.
[1] See,.e.g., D. Forster, Hydrodynamic Fluctuations, Broken Symmetry, and Correlation Functions, Benjamin (Reading, MA 1975).

[2] T. Moriya, Spin Fluctuations in Itinerant Electron Magnetism, Springer (Berlin 1985).

[3] T. R. Kirkpatrick and D. Belitz, Phys. Rev. B 53, 14364 (1996).

[4] T. Vojta, D. Belitz, R. Narayanan, and T. R. Kirkpatrick, Z. Phys.B 103, 451 (1996).

[5] By 'weak localization' we mean the nonanalytic behavior of electronic correlation functions in the limit of zero momentum and/or frequency that is induced by quenched disorder, or by a combination of interactions and disorder. For a review, see, e.g., P. A. Lee and T. V. Ramakrishnan, Rev. Mod. Phys. 57, 287 (1985).

[6] See, e.g., G. Baym and C. Pethick, Landau Fermi-Liquid Theory, John Wiley (New York 1991).

[7] D. Belitz and T. R. Kirkpatrick, Phys. Rev B 56, 6513 (1997).

[8] D. Belitz, T. R. Kirkpatrick, and T. Vojta, Phys. Rev. B 55, 9452 (1997).

[9] The universal long-wavelength, small-frequency effects we are interested in are independent of the microscopic details of the model used.

[10] J. A. Hertz, Phys. Rev. B 14, 1165 (1976).

[11] J. Zinn-Justin, Quantum Field Theory and Critical Phenomena, Clarendon Press (Oxford 1989).

[12] Renormalization effects generate a nonvanishing spin triplet interaction amplitude $\Gamma_{t}^{(\mathrm{ref})}$ in the reference ensemble, even though $\Gamma_{t}=0$ in the bare reference action. The reference enseble is thus a fully interacting electron system. The only restriction is that $\Gamma_{t}^{(\mathrm{ref})}$ must not be large enough to trigger a phase transition in the reference ensemble, lest the separation of scales that is crucial for our approach break down.

[13] It is important that even the longitudinal spin susceptibility of electrons in a magnetic field shows no nonanalytic wavenumber dependence of the type found in zero magnetic field, even though the soft mode structure would in principle allow for one; see D. Belitz and T. R. Kirkpatrick, Rev. Mod. Phys. 66, 261 (1994), Sec. V.A.2 for the disordered case, and T. Vojta (unpublished) for the clean one. The cancellations governed by these Ward identities reflect the fact that the vertices of the exact effective $\boldsymbol{\pi}$-field theory are still transverse correlation functions in some (complicated) reference system. Rotational invariance of the spins in this reference ensemble then ensures the softness of the transverse modes in the same way as it is ensured in the simple reference system $S_{0}^{\prime}$ that corresponds to our saddle-point approximation for the $\rho$-fluctuations.

[14] For a review, see, D. Belitz and T.R. Kirkpatrick, Rev. Mod. Phys. 66, 261 (1994).

[15] T. Moriya, Ref. [2], p.206 and Table 7.1. 